\title{
Gründe, Bedingungen und Formen von Rechtsrezeptionen
}

(vorgetragen in der Plenarsitzung am 29. Mai 2009)

\author{
Christian Starck
}

\section{Zum Begriff der Rezeption}

Die Rechtsrezeption gehört zu den umfassenden kulturellen Rezeptionsphänomenen, die zu allen Zeiten und in allen Kultursparten vorkommen. Es werden bestimmte soziale, geistige oder technische Entwicklungen oder Errungenschaften übernommen und in eine neue Umgebung eingefügt. Während die Rezeption etwa von Sprache oder kulturellen Gütern, von Literatur, Musik und bildender Kunst, aber auch der Mode, weitgehend formlos durch Individuen vonstatten geht, allerdings im Laufe der Zeit durch gesellschaftliche Anerkennung erst eigentlich verwirklicht ist, sind die Rechtsrezeptionen förmlich. Sie müssen es sein, weil Rechtsgeltung Form und Verfahren voraussetzt. Darüber wird noch besonders zu sprechen sein.

Rezeption ist ein Großthema auch innerhalb der Rechtswissenschaft. Mit dem Begriff Rechtsrezeption, um die es im folgenden geht, verbindet man in Europa, besonders in Deutschland, vor allem die Rezeption des römischen Rechts, einen Prozeß, der sich vom späten 13. bis zur Mitte des 16. Jahrhunderts erstreckte. Die Rezeption des römischen Rechts verdrängte das ältere deutsche Privatrecht zugunsten des im Corpus Iuris Civilis geordneten römischen Rechts in der privatrechtlichen Rechtslehre, Gesetzgebung und Rechtsanwendung. ${ }^{1}$ Der Vorgang ist für die europäische und wiederum besonders die deutsche Rechtskultur von großer Bedeutung gewesen $^{2}$, so groß, daß Heinrich Mitteis den Rezeptionsbegriff auf diesen

1 Franz Wieacker, Privatrechtsgeschichte der Neuzeit, 2. Aufl. 1967, S. 124 ff.; Georg Dahm, Zur Rezeption des römischen italienischen Rechts, Sonderausgabe 1960, S. 7 ff.; neuerdings sehr informativ Wolfgang Sellert, Zur Rezeption des römischen und kanonischen Rechts in Deutschland von den Anfängen bis zum Beginn der frühen Neuzeit: Überblick, Diskussionsstand und Ergebnisse, in: H.Boockmann/L.Grenzmann/B.Moeller/M.Staehelin (Hrsg.), Recht und Verfassung im Übergang vom Mittelalter zur Neuzeit, 1998, S. 115-166.

2 Paul Koschaker, Europa und das römische Recht, 3. Aufl. 1956; Reinhard Zimmermann, Römisches Recht und europäische Kultur, in: Juristenzeitung, 2007, S. 1-12. 
Vorgang beschränken wollte ${ }^{3}$. Ohne Erfolg! Rezeption ist das von recipere abgeleitete Substantiv. Recipere bezeichnet den aktiven Vorgang von aufnehmen, annehmen, entgegennehmen. Das weströmische Reich war untergegangen, Ostrom war schwach und stand kurz vor dem Untergang. Das im 6. Jahrhundert von den Justinianischen Juristen im Corpus Iuris Civilis gesammelte römische Recht war nicht das Recht eines Eroberungsvolkes, das sein Recht den unterworfenen Völkern oktroyiert, sondern totes Recht erlebte eine „Wiedergeburt durch Rezeption“.4

Ich möchte den Rezeptionsbegriff in seiner ursprünglichen sprachlichen Bedeutung verstehen. Deshalb werde ich nicht behandeln unerbetene Rechtsübertragungen von Imperialmächten. Darunter fällt der Rechtsoktroi durch die Sowjetunion in Mittel- und Osteuropa. Aber auch die Einführung des Common Law in den englischen Kolonien, einschließlich Nordamerika, ist keine Rezeption. Die englischen Kolonisten haben ihr Recht mitgebracht, nach dem sie leben wollten. Die Kolonisierungen in Afrika und Asien waren auch Kolonisierung durch Recht, das verliehen, verpflichtend gemacht, oktroyiert (abgeleitet von auctorare) wurde. Die kolonisierten Völker wurden passiv dem fremden Recht durch königliche Erlasse oder Verordnungen unterworfen; sie haben das fremde Recht nicht „angenommen“. Mit den Kolonialbeamten kamen englische Kolonialrichter. Die Verfestigung des oktroyierten Common Law fand durch die Ausbildung von Eingeborenen an den englischen Rechtsschulen statt. Die zu Richtern ausgebildeten Eingeborenen sprechen heute unter Schafswollperücken Recht. Aus heutiger Sicht spricht man hinsichtlich des ursprünglich oktroyierten Common Law mit einem gewissen Recht von Rezeption. ${ }^{5}$ Denn die ehemaligen Kolonien haben oktroyiertes Recht inzwischen „angenommen", soweit sie es nicht abgestoßen haben.

Wenn man den Rechtstext zur Grundlage der Rezeption nimmt, bedeutet das nicht, daß das Recht wie ein stofflicher Gegenstand aufgenommen wird und erstarrt in neuer sozialer Ambiance gilt. Alle Rezeptionsprozesse setzen mehr oder minder Assimilationen voraus, die zumeist beidseitig sind. Recht läßt sich nicht wie eine Pflanze transplantieren. Als geistiges Phänomen muß es von der einen in die andere Rechtsordnung eingepaßt werden. Das gilt vor allem, wenn einzelne Normen oder Normenkomplexe (= Institutionen) übernommen werden. Das rezipierte Recht muß

3 Heinrich Mitteis, Die Rechtsgeschichte und das Problem der historischen Kontinuität, Abhandlungen der Deutschen Akademie der Wissenschaften, Berlin 1947.

4 So Heinrich Henkel, Einführung in die Rechtsphilosophie, 2. Aufl. 1977, S. 219.

5 Konrad Zweigert/Hein Kötz, Einführung in die Rechtsvergleichung, 3. Aufl. 1996, S. 220 ff., $224 \mathrm{ff}$. 
in den rechtsdogmatischen und den gesellschaftlichen Zusammenhang des übernehmenden Staates gebracht werden, wenn die Rezeption auf Dauer gelingen soll.

\section{Gründe und Bedingungen für Rezeptionen}

1. Ubi societas, ibi ius, d.h. wo Menschen zusammenleben, gilt Recht. Eine Rezeption trifft also nie auf einen rechtlosen Zustand. Deshalb erhebt sich die Frage, welche Gründe es sind, die zur Rezeption führen: zur Rezeption ganzer Rechtsgebiete, einzelner Institutionen oder einzelner Rechtssätze. Man wird dies am besten an Hand ausgewählter historischer Beispiele verdeutlichen können. Ursachen können sein die Unvollkommenheit der eigenen Rechtsordnung, etwa Rechtszersplitterung, lang anhaltende Diktatur, die überwunden wird, oder der Wunsch der Teilhabe an rechtsdogmatisch ausgearbeitetem, entwickeltem „modernen“ Recht. Mit den Gründen für den Rezeptionsvorgang sind die Bedingungen eng verbunden, die gegeben sein müssen, damit die Rezeption die gewünschten Früchte trägt.

2. Ich habe hier vor einigen Jahren über deutsches Verfassungsrecht in Japan vorgetragen (Jahrbuch 2005, S. 95ff.). Japan ist ein gutes Beispiel für die Rechtsrezeption vor allem aus Deutschland. Japan erkannte, daß es wegen seiner traditionellen Isolation rückständig war, schickte Juristen zum Studium des Rechts nach Europa und reformierte sein Rechtssystem auch mit Hilfe deutscher Juristen in Japan. Der Vorgang war 1898 mit Inkrafttreten des japanischen BGB abgeschlossen. In China bestanden die gleichen Gründe für Rechtsrezeptionen. Nach dem Motto „Von Japan lernen heißt siegen lernen" sind in den letzten acht Jahren der Qing-Dynastie (1903-1911) Gesetzentwürfe und Kodifikationsprojekte nach westlichem, vor allem deutschem Muster verfaßt worden. ${ }^{6}$ Da China nach dem Sturz des Kaisertums keine feste staatliche Form ausbilden konnte, fehlten die Bedingungen für die Rezeption. Dagegen nahm Taiwan, das China im Friedensvertrag von Shimonoseki (1895) an Japan hatte abtreten müssen und das nun japanische Provinz geworden war, an der in Japan stattfindenden Rezeption europäischen, insbesondere deutschen Rechts teil, hat also einen bis heute wirkenden Modernisierungsvorteil gegenüber China.

$6 \quad$ Dazu ausführlich Robert Heuser, Beginn eines Jahrhundertprojekts: Die Rechtsreform unter der späten Qing-Dynastie (1903-1911), in: Zeitschrift für chinesisches Recht, 15. Jg., 2008, S. 193-205. 
3. Beim Übergang von der Diktatur zum demokratischen Verfassungsstaat haben die meisten Länder Verfassungsgerichte errichtet. Ein Verfassungsgericht soll die Einhaltung der Verfassung, d.h. insbesondere der Kompetenzordnung und der Grundrechte, garantieren und damit die Normativität der Verfassung sichern. Aus dieser Aufgabenstellung für Verfassungsgerichte erklärt sich der Aufschwung, den die Verfassungsgerichtsbarkeit zunächst in Westeuropa nach dem Zweiten Weltkrieg erlebte. Die neu oder wiedererrichtete rechtsstaatliche Ordnung sollte institutionell gesichert werden. Die sichtbar guten Erfahrungen, die die Bundesrepublik Deutschland mit dem Bundesverfassungsgericht gemacht hat, waren der Hauptgrund für die Einrichtung der Verfassungsgerichtsbarkeit in Spanien nach der Überwindung der Diktatur.

In vielem ähnelt das spanische Verfassungsgericht dem deutschen Bundesverfassungsgericht, vor allem durch die Zuständigkeit für Urteilsverfassungsbeschwerden. Die Rezeption dieser bei spezialisierten Verfassungsgerichten äußerst selten vorkommenden Zuständigkeit muß man vor dem Hintergrund sehen, daß bei der Transición zum demokratischen Verfassungsstaat mit der Verfassung von 1978 das Oberste Gericht in seiner Zusammensetzung nicht angetastet wurde. Mit der Urteilsverfassungsbeschwerde vor dem neu errichteten Verfassungsgericht war die Möglichkeit gegeben, die Entscheidungen des Obersten Gerichts auf ihre Vereinbarkeit mit den Grundrechten zu prüfen, die in der neuen Verfassung nicht nur verzeichnet waren, sondern effektiv gelten sollen (Art. 53 Span. Verf.).

Eine Parallele zu der in Spanien eingeführten Urteilsverfassungsbeschwerde finden wir 15 Jahre später in Südafrika. Bei der Ablösung des Apartheidsystems blieb die Zusammensetzung der Gerichte unangetastet. Entgegen der in Südafrika herrschenden Common Law-Tradition wurde ein spezialisiertes Verfassungsgericht eingerichtet. Bei dieser Hinwendung zum österreichisch-deutschen Modell der Verfassungsgerichtsbarkeit ${ }^{7}$ stand der Wille Pate, Einfluß auf letztinstanzliche Urteile der Gerichte zu nehmen. ${ }^{8}$ Es wurde darauf hingewiesen, daß die Gerichte in ihrer fortbestehenden Besetzung aus der Zeit der Apartheid zu lange Zeit brauchen würden, sich den Erfordernissen einer grundrechtsorientierten Auslegung anzupassen. ${ }^{9}$ Die Befürworter eines besonderen Verfassungsgerichts für Südafrika, die sich durchgesetzt haben, argumentierten mit der vorbildlichen Rechtsprechung des Bundesverfassungsgerichts zur Festigung des Grundrechts-

\footnotetext{
Jörg Fedke, Die Rezeption von Verfassungsrecht. Südafrika 1993-1996, 2000, S. 392.

8 Vgl. Art. 167 Abs. 6 lit. b der Südafrikanischen Verfassung: „must allow a person [... ] to appeal directly to the Constitutional Court from any other court".

9 Siehe mit Nachweisen Levin Holle, Das Verfassungsgericht der Republik Südafrika, 1997, S. 50.
} 
schutzes und bemerkenswerter Weise auch mit dessen zentraler Rolle für die Stabilisierung der neuen Rechtsordnung ${ }^{10}$ und damit des gesellschaftlichen Grundkonsenses. ${ }^{11}$

Die Verfassungsgerichtsbarkeit bietet auch ein Beispiel für Rezeptionswechsel. Die meisten südamerikanischen Staaten hatten von den USA die diffuse Verfassungsgerichtsbarkeit durch die ordentlichen Gerichte, vor allem durch das Oberste Gericht, rezipiert. Nach der Errichtung des spanischen Tribunal Constitucional im Jahre 1978 sind zahlreiche südamerikanische Staaten zur konzentrierten und spezialisierten Verfassungsgerichtsbarkeit durch ein Verfassungsgericht übergegangen und haben sich insoweit dem europäischen System angeschlossen ${ }^{12}$.

4. Mit der Verfassungsgerichtsbarkeit, die die Kompetenz hat, Gesetze auf ihre Verfassungsmäßigkeit zu überprüfen, wird das Verhältnismäßigkeitsprinzip mit rezipiert. Das ist ein rechtsdogmatisches Instrument, mit dem man prüfen kann, ob die in Frage stehende Grundrechtsbegrenzung verfassungsmäßig ist oder das Grundrecht verletzt. Dafür wird geprüft, ob die Grundrechtseinschränkung geeignet ist, den Schutz des Rechtsguts zu bewirken. Sie muß außerdem erforderlich sein, was nicht der Fall ist, wenn ein milderes Mittel ausreicht. Außerdem muß sie in angemessenem Verhältnis zu dem Gewicht und der Bedeutung des Grundrechts stehen. Die Rechtsprechung des Bundesverfassungsgerichts zur Einschränkung von Grundrechten ist vom EGMR übernommen worden und hat direkt oder vermittelt über den EGMR auf Österreich, Italien und auf Spanien, Portugal, Polen etc. gewirkt.

5. Zusammenfassung. Aus den bisherigen Darlegungen lassen sich zahlreiche Gründe für Rezeptionsvorgänge extrahieren: Bewährte, als vorbildlich empfundene Rechtsinstitutionen oder Einzelregelungen werden übernommen, um fehlende oder schlechte eigene Regelungen zu kompensieren und gleichzeitig am erkennbaren Fortschritt anderer Rechtsordnungen teilzuhaben. Bei der politischen Überwindung von Diktaturen werden die neue Ordnung stabilisierende Institutionen rezipiert, die sich in demokratischen Verfassungsstaaten bewährt haben. Ein Übergang von der diffusen Ver-

10 Vgl. im einzelnen Marinus Wiechers, A Constitutional Court for South Africa, 1991, S. 292; Kader Asmal, Constitutional Courts - a comparative survey, in: The Comparative and International Law Journal of South Africa, 1991, S. 320.

11 Christian Starck, Freiheit und Institutionen, 2002, S. 319, $329 \mathrm{ff}$.

12 Siehe dazu ausführlich allgemein und speziell für jedes lateinamerikanische Land Norbert Lösing, Die Verfassungsgerichtsbarkeit in Lateinamerika, 2001, S. 28 ff., 37 f.; Allan R. Brewer Carias, La jurisdicción constitucional en America Latina, in: García Belaunde/Fernández Segado (coord.), La jurisdicción constitucional en Iberoamerica, Madrid 1997, S. 141 ff. 
fassungsgerichtsbarkeit durch die ordentlichen Gerichte zur spezialisierten Verfassungsgerichtsbarkeit, für die ein neues Verfassungsgericht eingerichtet wird, findet z.B. statt, um einer neuen Verfassung normative Wirkung zu garantieren, ohne die aus dem alten System stammenden Richter austauschen zu müssen.

\section{Formen der Rezeption}

1. Am deutlichsten erkennbar sind Rezeptionen durch Verfassungs- und Gesetzgebung. Fremde Normen werden in die Sprache des rezipierenden Staates übersetzt und in die Rechtsordnung eingefügt. Der neue Zusammenhang, in dem sie stehen, verleiht ihnen eventuell eine Bedeutungsnuance. Auch die gesellschaftliche Ambiance mag eine Rolle spielen. Verfassunggebung oder Gesetzgebung ist aber erst der Beginn der Rezeption. Es kommt in der Folgezeit darauf an, wie die Rechtsprechung die rezipierten Normen auslegt und anwendet und ob die Rechtsdogmatik durch die gelehrten Juristen mit der Rezeption angemessen umzugehen versteht. Mögen die Gründe für die Rezeption bei der gesetzlichen Inkorporation dargelegt worden sein, so ist damit noch nicht gesichert, daß günstige Bedingungen für das Gelingen der Rezeption herrschen. Die dargestellten Beispiele zeigen durchgehend, daß Rezeptionen mittels Verfassung- und Gesetzgebung durch die Rechtslehrer vorbereitet werden, die das fremde Recht zunächst studieren, wie es konstruiert ist, wie es wirkt und wie es im eigenen Land wirken könnte. Was Portugal und Spanien betrifft, waren günstige Bedingungen für die geschilderten und weitere Rezeptionen, daß noch während der Diktaturen zahlreiche junge Juristen ihre heimischen Studien in Deutschland fortsetzten und später das rezipierte Recht somit für sie kein fremdes Recht war. Sieht man sich die personelle Zusammensetzung des portugiesischen und des spanischen Verfassungsgerichts besonders in den ersten Perioden an, so stellt man fest, daß viele Richter, auch Präsidenten ${ }^{13}$, das deutsche Verfassungsrecht aus eigenen Studien in Deutschland kannten, zumeist als Humboldt-Stipendiaten. Dasselbe gilt für Polen, Ungarn etc.

2. Die Rechtsprechung ist aber auch autochthone Form der Rezeption, d.h. sie initiiert Rechtsrezeptionen ohne vorgängige Gesetzgebung. Ein eindrucksvolles Beispiel für dieses Phänomen stellt die Rechtsprechung des polnischen Verfassungsgerichts in der Zeit nach Beendigung der kommu-

13 José Manuel Moreira Cardosa da Costa (Portugal), Pedro Cruz Villalón (Spanien). 
nistischen Diktatur (1989) und vor dem Erlaß der neuen Verfassung (1997) dar. In der Zwischenzeit hat das polnische Verfassungsgericht die alte Verfassung, die von der Klausel über die Souveränität der Vereinigten Arbeiterpartei befreit worden war, in Riesenschritten durch Rezeptionen vor allem aus der Menschenrechtskonvention in Richtung auf eine rechtsstaatliche Verfassung fortgebildet ${ }^{14}$ und damit der Verfassung von 1997 vorgearbeitet.

3. Eine dritte Form der Rezeption ist die Übernahme fremden Rechts durch die Rechtslehre mit Spätwirkungen auf Rechtsprechung und Gesetzgebung. So hat Otto Mayer Ende des 19. Jahrhunderts sein Werk „Theorie des französischen Verwaltungsrechts“ (1886), das hauptsächlich vom Conseil d'Etat gebildet worden ist, geschrieben und dieses später rezipiert bei seiner maßgeblichen rechtsdogmatischen Bearbeitung des deutschen Verwaltungsrechts, ${ }^{15}$ auf das sich die deutsche Verwaltungsrechtsprechung stützte, die 80 Jahre nach Otto Mayer dann Grundlage für das Verwaltungsverfahrensgesetz (1976) war.

Die intensivste Form der Rezeption ist: Gesetz, Rechtsprechung, Rechtslehre. Alle arbeiten zusammen. Es beginnt mit dem Studium des Rechts in Deutschland, Lehrer aus Deutschland kommen in das rezeptionswillige Land, dessen Richter werden nach Deutschland geschickt. Das ist die perfekteste Form, die mir bekannt geworden ist. ${ }^{16}$ Dann kommt noch eine Übersetzung des führenden Kommentars zum rezipierten Recht hinzu, mehr kann man nicht machen.

Zum Schluß: Es gibt auch den Rezeptionswechsel durch Gesetzgebung. Als Hauptbeispiel dafür habe ich den Wechsel von der diffusen zur spezialisierten Verfassungsgerichtsbarkeit in einigen südamerikanischen Ländern genannt. Rezeptionswechsel bietet sich auch dann an, wenn trotz Assimilierungsversuchen die Rezeption gescheitet ist. Man lernt aus Fehlern. Die Assimilierung findet weitgehend durch Rechtsprechung statt. Der Richter ist derjenige, der die einzelnen konkreten Probleme aus seiner Beschäftigung mit den konkreten Fällen besser kennt als der Gesetzgeber. Der Gesetzgeber

14 Vgl. dazu Lech Garlicki, Constitutional Development in Poland, in: Saint Louís University Law Review, vol. 32 No. 3, 1998, S. 713 ff.; Piotr Tuleja, Grundlagen und Grundzüge staatlichen Verfassungsrechts: Polen, in: v. Bogdandy/Cruz Villalón/Huber, Handbuch des Ius Publicum Europaeum, Bd. I, 2007, S. 451, 462; Piotr Czarny, Rechtsrezeptionen im polnischen Verfassungsrecht durch Verfassunggebung und Verfassungsgericht, in: Heun/Starck/Tsai (Hrsg.), Rezeption und Paradigmenwechsel im öffentlichen Recht, 2009, S. $111 \mathrm{ff} ., 114 \mathrm{f}$.

15 Otto Mayer, Deutsches Verwaltungsrecht, 2 Bände, 1895 und 1896.

16 Vgl. dazu Feng-zhi Peng, Die Rezeption der deutschen Verwaltungsgerichtsordnung im taiwanesischen Verwaltungsprozeßgesetz, in: Heun/Starck/Tsai (Hrsg.), Rezeption und Paradigmenwechsel im öffentlichen Recht, 2009, S. 179, 181 ff. (Rezeption des Gesetzes), 186 ff. (Rezeption der Anwendung des Gesetzes). 
hat eine allgemeine Idee; wenn aber Fälle gelöst werden müssen, merkt man erst, wie weit die Idee trägt. Oft sind die Gesetze so allgemein formuliert, daß angemessene Fallösungen möglich sind, da ja Gesetzesanwendung Gesetzesauslegung voraussetzt, in der immer ein Stück Anpassung liegt. In diesem Zwischenfeld bewährt sich Rezeption oder nicht. 